\title{
Achilles tendon disease in lung transplant recipients: association with ciprofloxacin
}

\author{
P.N. Chhajed, M.L. Plit, P.M. Hopkins, M.A. Malouf, A.R. Glanville
}

Achilles tendon disease in lung transplant recipients: association with ciprofloxacin. P.N. Chhajed, M.L. Plit, P.M. Hopkins, M.A. Malouf, A.R. Glanville. (C)ERS Journals Ltd 2002.

ABSTRACT: Achilles tendonitis or rupture are uncommon complications following the use of fluoroquinolones, with a reported incidence in the general population of $0.4 \%$. The aims of the current study were to determine the incidence of Achilles tendon disease (ATD) in lung transplant recipients (LTR) and to identify risk factors.

Questionnaires were sent to 150 LTR of whom 101 responded (67\%). Twenty-two LTR (21.8\%) experienced ATD (tendonitis 16, rupture six). The mean age of LTR who developed ATD was 52.9 \pm 6.1 yrs (range: 19-63.5 yrs).

Only the use of ciprofloxacin was significantly associated with ATD $(\mathbf{p}<0.05)$. Age, sex, underlying disease necessitating transplantation, serum creatinine and cyclosporine levels were not associated with ATD. The association between ciprofloxacin and ATD was not dose related. Of the 72 LTR who had received ciprofloxacin, $20(28 \%)$ developed ATD (tendonitis 15, rupture five). In patients receiving ciprofloxacin, there was no association between the mean cumulative dose of prednisolone and ATD. Tendon rupture occurred with a lower ciprofloxacin dosage than tendonitis and the mean recovery duration was significantly longer.

To conclude, lung transplant recipients receiving ciprofloxacin are at significant risk of developing Achilles tendon disease. The association between ciprofloxacin and Achilles tendon disease appears to be idiosyncratic rather than dose-related.

Eur Respir J 2002; 19: 469-471.
Heart Lung Transplant Unit, St. Vincent's Hospital, Victoria Street, Darlinghurst, Sydney, NSW 2010, Australia.

Correspondence: P.N. Chhajed, Heart Lung Transplant Unit, St. Vincent's Hospital, deLacy Building, Level 14, Victoria Street, Darlinghurst, Sydney, NSW 2010, Australia.

Fax: 61293324267

E-mail: chhajed@hotmail.com

Keywords: Achilles tendon

ciprofloxacin

fluoroquinolane

lung transplant

tendonitis

tendon rupture

Received: June 292001

Accepted after revision September 17 2001
Achilles tendonitis or rupture are uncommon complications following the use of fluoroquinolones with a reported incidence in the general population of $0.4 \%$ [1]. Fluoroquinolone antibiotics implicated in Achilles tendon disease (ATD) include ofloxacin, pefloxacin and ciprofloxacin [1-4]. Supraspinatus and Achilles tendonitis have been reported in patients with renal transplantation, but an association with ciprofloxacin was not explored [5]. This study was undertaken in order to determine the incidence of ATD in lung transplant recipients (LTR) and to identify risk factors.

\section{Patients and methods}

Questionnaires were sent to all 150 living LTR who had undergone transplantation at the institute. The questionnaire inquired about a history of ankle pain, a diagnosis of Achilles tendonitis or rupture, time interval to resolution (if applicable) and ciprofloxacin usage. One-hundred and one LTR $(67.3 \%)$ responded to the questionnaire (male:female (M:F), 51:50; mean age $47.6 \pm 12.1$ yrs. The response rate was $89 \%$ (76 of 85 ) in patients being followed-up in the clinic and $38.4 \%$ in those who were followed-up in their local hospitals (e.g. interstate). None of the patients had symptoms or signs of polyarthritis, spinal disease, eye changes, skin changes, urethritis or any evidence of a seronegative polyarthritis syndrome. All patients received ciprofloxacin for treatment of lower respiratory tract infection, which was either due to pseudomonas $(n=68)$ or atypical mycobacteria $(n=4)$.

Achilles tendonitis was defined by the presence of pain over the tendon with walking or on palpation, and was usually associated with marked swelling [3]. The hallmark of Achilles tendon rupture is a positive Thompson's test [3]. All patients were treated with triple drug immunosuppression (cyclosporine/ tacrolimus, azathioprine and prednisolone) posttransplantation. The cumulative dose of prednisolone was calculated from the date of transplantation to the time ATD was noted. In all instances, ciprofloxacin was discontinued with the advent of this complication. The total dose of ciprofloxacin (g) taken during the event when ATD occurred was calculated, and the serum cyclosporine and serum creatinine levels recorded. In those LTR who had received ciprofloxacin and did not develop ATD, the cumulative dosage of prednisolone $(\mathrm{g})$ was calculated from the date of transplantation until the recipient had taken the last course of ciprofloxacin. The total dose of ciprofloxacin taken during the last course, serum cyclosporine and serum creatinine levels were also recorded. In the group of LTR who had not received ciprofloxacin, the last documented serum creatinine level was recorded. 
Age, sex, underlying disease and serum creatinine level were compared as independent risk factors for association with ATD.

In LTR who developed ATD, the mean duration from the day of transplant to the development of ATD was 810 \pm 991 days (range: 13-3,580 days). In LTR who did not develop ATD, the mean duration from the day of transplant to the day when these patients were last assessed was $1213 \pm 1025$ days (range: 2-3,927 days). There was a significant difference in these two periods $(p=0.0397)$ and hence the cumulative dosage of steroids in these groups was not compared. Serum cholesterol levels were not available in all patients and hence were not compared.

\section{Lung transplant recipients receiving ciprofloxacin}

The pre-morbid forced expiratory volume in one second in patients with ATD was $2.2 \pm 0.82 \mathrm{~L}$ and in those not developing the disease was $2.1 \pm 0.89 \mathrm{~L}$ $(p>0.05)$. There was no significant difference in the mean period from the day of transplantation to the development of ATD ( $860 \pm 1025$ days) or until the last documented course of ciprofloxacin in those who did not develop this complication ( $955 \pm 900$ days). This enabled the comparison of the cumulative dosage of prednisolone in these two groups of patients. The component of the cumulative dose of prednisolone, which was given pre-transplant, was not available.

\section{Statistical analysis}

Logistic regression was used to compare the association of ciprofloxacin, sex, age, underlying disease necessitating transplantation, postoperative day since transplantation and serum creatinine and cyclosporine levels with ATD. The Mann-Whitney U-test was used to determine the significance between two means.

\section{Results}

Of the 101 LTR who responded to the questionnaire, $22(21.8 \%)$ recipients (M:F, 13:9) had experienced ATD (tendonitis 16, rupture six). Ciprofloxacin was significantly associated with ATD $(p<0.05)$. Age, sex, underlying disease and serum creatinine and cyclosporine levels were not associated with ATD. There was a tendency for patients with emphysema to have ATD, however this did not reach statistical significance $(\mathrm{p}=0.07)$. Seventy-two recipients $(71.3 \%)$ had received ciprofloxacin at some stage following transplantation. Twenty LTR $(27.8 \%)$ reported the development of ATD (tendonitis 15, rupture five) following the use of ciprofloxacin. The remaining two patients who reported ATD (tendonitis one, rupture one) had not received ciprofloxacin following transplantation.

\section{Lung transplant recipients receiving ciprofloxacin}

The association between ciprofloxacin and ATD was not dose related. The mean total dose of ciprofloxacin in LTR developing ATD (17.6 $\pm 12.7 \mathrm{~g}$; range: 3-63 g) was significantly lower than the dose received by those not developing the disease (135.2 \pm $313.7 \mathrm{~g} ; 4-1,895 \mathrm{~g})(\mathrm{p}<0.05)$. The mean cumulative dose of prednisolone in patients developing ATD was $15.1 \pm 11.2 \mathrm{~g}(2.1-33.2 \mathrm{~g})$, and in those not developing the disease was $16.3 \pm 12.1 \mathrm{~g}$, (1.4-46.6 g; $\mathrm{p}>0.05)$. The mean duration of onset of ATD after ciprofloxacin was $10.8 \pm 5$ days (2-20 days) and the mean recovery time was $29.5 \pm 39.4$ weeks (2-180 weeks). The recovery time was significantly earlier in patients with tendonitis (17.5 \pm 14.4 weeks; 2-60 weeks) compared to patients having tendon rupture (65.6土 66.9 weeks; $8-180$ weeks; $\mathrm{p}<0.05$ ). The cumulative dosage of ciprofloxacin was significantly lower in patients with tendon rupture $(10.7 \pm 3 \mathrm{~g} ; 7-14 \mathrm{~g})$ compared to patients having tendonitis $(19.9 \pm 13.9 \mathrm{~g}$; $3-63 \mathrm{~g} ; \mathrm{p}<0.05)$.

Achilles tendon disease in lung transplant recipients not associated with ciprofloxacin

One LTR developed spontaneous Achilles tendonitis on day 49 post-transplantation and the other developed spontaneous Achilles tendon rupture on day 573 post-transplantation. Recovery in the patient with tendonitis took 8 weeks and the patient with tendon rupture still has some ankle pain almost 4-yrs later.

\section{Discussion}

Tendons are connective tissue structures with a low cell content, mainly fibroblasts, little vascularity, and are primarily composed of collagen fibres [6]. The diminishing vascularity of the Achilles tendon with advancing age [7] and steroid use [8] has been demonstrated. JORGENSEN et al. [9] proposed that tendon rupture might be due to a vascular phenomenon leading to ischaemia. To the best of the authors' knowledge there are no published data regarding ATD in LTR. The overall incidence of ATD in this study was $21.8 \%$. The reported incidence of ATD in the general population associated with the use of fluoroquinolone antibiotics is $0.4 \%$ [1]. The incidence of tendonitis in this study $(16 \%, 16$ of 101$)$ compares with a report in renal transplantation $(20 \%)$, however, ciprofloxacin was not analysed as a risk factor in that study [5]. In the present study, ATD was significantly associated with the use of ciprofloxacin. Long-term steroid therapy has been reported as an independent risk factor as well as in association with the use of fluoroquinolones for the development of ATD [10,11]. All patients in this study received prednisolone as part of their immunosuppression strategy following lung transplantation. Steroids delay the maturation of fibroblasts and reduce the tensile strength of the tendon [11-13]. Although the cumulative dose of prednisolone did not appear to be a risk factor for the development of ATD in the LTR receiving ciprofloxacin, the possibility that prednisolone does play an important role cannot be excluded. LTR have 
considerable exposure to prednisolone pre- and posttransplantation compared to the general population whose exposure to long-term steroids is minimal.

In the present study, ATD occurred at a mean duration of 10.8 days following the administration of ciprofloxacin and this is consistent with the literature, which reports an onset within the first 2 weeks $[1,2$, 10]. The ratio of Achilles tendonitis to Achilles tendon rupture was $4: 1$, and this was similar to the series reported by ROYER et al. [2]. In general, ATD heals slowly and when tendon rupture occurs, healing time is considerably lengthened. Delays in healing of 1-3 months for tendonitis and 1-6 months for tendon rupture have been reported [3,10,14,15]. Advanced age in association with fluoroquinolone use has been reported as a risk factor for ATD [10,16]. In this study, age was not identified to be a risk factor, however, the patients who developed ATD were $\geqslant 35 \mathrm{yrs}$ in age. The recovery times for ciprofloxacin-associated tendonitis and rupture were much higher in this study. This may be due to the effect of prednisolone.

The patients in this study received a wide range of total ciprofloxacin dose $(3-1,895 \mathrm{~g})$. The mean total dose of ciprofloxacin in LTR developing ATD was significantly lower compared to those not developing the disease. The mean total ciprofloxacin dose was significantly higher in LTR with tendonitis compared to tendon rupture. These findings support the conclusion that ciprofloxacin associated ATD in LTR is not dose-related and favours an idiosyncratic mechanism of action on the Achilles tendon. There was a tendency for patients with emphysema to have ATD, however this did not reach statistical significance. The likely exposure of this group of patients to significant dosages of prednisolone pre-transplant cannot be excluded.

VAN DER LINDEN et al. [1] found no correlation between renal dysfunction and tendonitis, whereas, Achilles tendonitis and other tendon ruptures have been reported as having a greater incidence in patients with end-stage renal disease [5] and those receiving haemodialysis [17-19]. It has been postulated that chronic acidosis in dialysis patients leads to degeneration of tendons, thereby causing a change in their tensile characteristics [18]. In the current study, there was no correlation between ATD and serum creatinine levels.

In conclusion, the present study suggests that lung transplant recipients who receive ciprofloxacin are at significant risk of developing Achilles tendon disease. The overall association of ciprofloxacin and Achilles tendon disease is not dose related. Tendon rupture occurs at a lower total dose of ciprofloxacin than tendonitis emphasizing idiosyncratic susceptibility to Achilles tendon disease. Ciprofloxacin remains an important antibiotic in the armamentarium of outpatient treatment of pseudomonas infection. However, lung transplant recipients should be aware of this potential complication in order to facilitate early recognition, and discontinuation of this medication.

\section{References}

1. van der Linden PD, van de Lei J, Nab HW, Stricker $\mathrm{BH}$. Achilles tendinitis associated with fluoroquinolones. Br J Clin Pharmacol 1999; 48: 433-437.

2. Royer RJ, Pierfitte C, Netter P. Features of tendon disorders with fluoroquinolones. Therapie 1994; 49: 75-76.

3. Harrell RM. Fluoroquinolone-induced tendinopathy: what do we know? South Med J 1999; 92: 622-625.

4. Movin T, Gad A, Guntner P, Foldhazy Z, Rolf C. Pathology of the Achilles tendon in association with ciprofloxacin treatment. Foot Ankle Int 1997; 18: 297 299.

5. Murison MS, Eardley I, Slapak M. Tendinitis - a common complication after renal transplantation. Transplantation 1989; 48: 587-589.

6. Le Huec JC, Schaeverbeke T, Chauveaux D, Rivel J, Dehais J, Le Reteller A. Epicondylitis after treatment with fluoroquinolone antibiotics. J Bone Joint Surg Br 1995; 77: 293-295.

7. Langergren C, Lindholm A. Vascular distribution of Achilles tendon: an angiographic and microangiographic study. Acta Chir Scand 1958/59; 116: 491.

8. Lund IM, Donde R, Knudsen EA. Persistent local cutaneous atrophy following corticosteroid injection for tendinitis. Rheumatol Rehabil 1979; 18: 91-93.

9. Jorgensen C, Anaya JM, Didry C, et al. Arthropathy with achilles tendon involvement induced by pefloxacin. Apropos of a case. Rev Rhum Mal Osteoartic 1991; 58: 623-625.

10. Zabraniecki L, Negrier I, Vergne P, et al. Fluoroquinolone induced tendinopathy: report of 6 cases. J Rheumatol 1996; 23: 516-520.

11. Haines JF. Bilateral rupture of the Achilles tendon in patients on steroid therapy. Ann Rheum Dis 1983; 42: 652-654

12. Kennedy JC, Willis RB. The effects of local steroid injections on tendons: a biomechanical and microscopic correlative study. Am J Sports Med 1976; 4: 1121.

13. Wrenn RN, Goldner JL, Markee JL. An experimental study of the effect of cortisone on the healing process and tensile strength of tendons. J Bone Joint Surg 1954; 36A: 588.

14. McEwan SR, Davey PG. Ciprofloxacin and tenosynovitis. Lancet 1988; 2: 900.

15. Perrot S, Kaplan G, Ziza JM. 3 cases of Achilles tendinitis caused by pefloxacin, 2 of them with tendon rupture. Rev Rhum Mal Osteoartic 1992; 59: 162.

16. Szarfman A, Chen M, Blum MD. More on fluoroquinolone antibiotics and tendon rupture. $N$ Engl J Med 1995; 332: 193.

17. Morein G, Goldschmidt Z, Pauker M, Seelfreund M, Rosenfeld JB, Fried A. Spontaneous tendon ruptures in patients treated by chronic haemodialysis. Clin Orthop 1977; 124: 209-213.

18. Lotem M, Robson MD, Rosenfield JB. Spontaneous rupture of the quadriceps tendon in patients on chronic haemodialysis. Ann Rheum Dis 1974; 33: 428-429.

19. Murphy KJ, McPhee J. Tear of major tendons in chronic acidosis with elastosis. J Bone Joint Surg 1965; 47A: 1253. 